\title{
I 2.4 ACZ885 (canakinumab), a new IL-I beta blocking monoclonal antibody provides long-lasting remission in children with cryopyrin associated periodic syndrome (CAPS)
}

\author{
J Kümmerle-Deschner*1, N Blank², J Roesler ${ }^{3}$, E Ramos ${ }^{4}$, N Tzaribachev ${ }^{1}$, \\ K Gramlich $^{1}$, C Well ${ }^{1}$, SD Felix ${ }^{5}$, C Rordorf ${ }^{5}$ and T Jung ${ }^{5}$
}

Address: ${ }^{1}$ Universitätsklinik Tübingen, Kinderklinik I, Hoppe-Seyler-Straße 1, Tübingen, Germany, ${ }^{2}$ Medizinische Klinik 5, Im Neuenheimer Feld 410, Heidelberg, Germany, ${ }^{3}$ Univ.-Klinikum Carl-Gustav-Carus, Kinderklinik, Fetscherstraße 74, Dresden, Germany, ${ }^{4}$ Servicio de Pediatria, Hospital Central de Asturias, C/Julian Claveria, s/n, Oviedo, Spain and ${ }^{5}$ Novartis Pharma AG, Basel, Switzerland

* Corresponding author

from $15^{\text {th }}$ Paediatric Rheumatology European Society (PreS) Congress

London, UK. 14-17 September 2008

Published: 15 September 2008

Pediatric Rheumatology 2008, 6(Suppl I):S26 doi:I0.I I86/I546-0096-6-SI-S26

This abstract is available from: http://www.ped-rheum.com/content/6/SI/S26

(c) 2008 Kümmerle-Deschner et al; licensee BioMed Central Ltd.

This study was conducted to assess the efficacy, safety, and immunogenicity of canakinumab administered as a subcutaneous injection to patients with CAPS. Herein we report the results of 5 children (4-13 years) and 2 adolescents (16 and 17 years) enrolled in the study. All had documented NALP-3 gene mutations. Entry criteria were active disease evaluated by moderate to severe symptoms of CAPS (based on a 5 point global physician's assessment scale) and/or elevated CRP and SAA levels. The children received canakinumab $2 \mathrm{mg} / \mathrm{kg}$, while the 2 adolescents received a dose of $150 \mathrm{mg}$. Subsequent injections were administered after each relapse. Clinical remission was defined as: physicians global assessment of disease activity absent/minimal, assessment of skin disease absent/ minimal, normal serum SAA and CRP levels. Canakinumab improved symptoms in the majority of patients within 1 day, with full clinical remission within 7 days. Two children received subsequent additional intravenous injections $(5 \mathrm{mg} / \mathrm{kg})$ to achieve a complete response. The median time to re-dosing due to relapse was 88 days $(n=$ $6)$ and 63 days $(n=5)$ after the first and second dose of canakinumab respectively. One patient was discontinued 70 days after the first dose due to pregnancy. Injections were well tolerated and so far no immunogenicity developed. The total exposure time ranged from 70 to 383 days. Adverse events (AE) were mainly upper respiratory tract infections. One serious AE was reported (vertigo). In con- clusion, canakinumab was well tolerated and provided long-lasting complete clinical remission in children with CAPS. 